\title{
Enterococcus hirae bacteremia associated with acute pyelonephritis in a patient with alcoholic cirrhosis: a case report and literature review
}

Tomoaki Nakamura ${ }^{1 *}$ (D), Kazuhiro Ishikawa', Takahiro Matsuo ${ }^{1}$, Fujimi Kawai ${ }^{2}$, Yuki Uehara ${ }^{1,3,4,5}$ and Nobuyoshi Mori ${ }^{1}$

\begin{abstract}
Background: Infections caused by Enterococcus hirae are common in animals, with instances of transmission to humans being rare. Further, few cases have been reported in humans because of the difficulty in identifying the bacteria. Herein, we report a case of pyelonephritis caused by E. hirae bacteremia and conduct a literature review on $E$. hirae bacteremia.

Case presentation: A 57-year-old male patient with alcoholic cirrhosis and neurogenic bladder presented with fever and chills that had persisted for 3 days. Physical examination revealed tenderness of the right costovertebral angle. Matrix-assisted laser desorption ionization-time of flight mass spectrometry (MALDI-TOF MS) of the patient's blood and urine samples revealed the presence of $E$. hirae, and pyelonephritis was diagnosed. The patient was treated successfully with intravenous ampicillin followed by oral linezolid for a total of three weeks.

Conclusion: The literature review we conducted revealed that $E$. hirae bacteremia is frequently reported in urinary tract infections, biliary tract infections, and infective endocarditis and is more likely to occur in patients with diabetes, liver cirrhosis, and chronic kidney disease. However, mortality is not common because of the high antimicrobial susceptibility of E. hirae. With the advancements in MALDI-TOF MS, the number of reports of E. hirae infections has also increased, and clinicians need to consider E. hirae as a possible causative pathogen of urinary tract infections in patients with known risk factors.
\end{abstract}

Keywords: Enterococcus hirae, Urinary tract infection, Alcoholic cirrhosis, Case report

\section{Background}

Enterococcus hirae primarily causes zoonosis $[1,2]$, with human infections being relatively rare. Nevertheless, pyelonephritis [3-5], infective endocarditis [6-11], and biliary tract infections $[5,12]$ due to $E$. hirae have been reported in human patients. Although $E$. hirae has been

\footnotetext{
*Correspondence: tonaka@luke.ac.jp

1 Department of Infectious Diseases, St. Luke's International Hospital, 9-1, Akashi-cho, Chuo-ku, Tokyo, Japan

Full list of author information is available at the end of the article
}

found to cause these severe diseases in humans, few cases have been reported because of the difficulty in identifying the bacteria, and the lack of comprehensive reports on clinical characteristics and treatments [3].

Matrix-assisted laser desorption ionization-time of flight mass spectrometry (MALDI-TOF MS) has recently emerged as an important diagnostic tool, characterized by its high speed, ease of use, and low per sample cost compared to those of conventional diagnostic tools [13]. Therefore, greater progress in the analysis of a variety of 
bacterial species that have been difficult to identify in the past is expected [13]. In a case of urinary tract infection, E. hirae was rapidly and correctly identified using MALDI-TOF MS, without any complementary tests [14]. Here, we report a case of bacteremia secondary to pyelonephritis caused by E. hirae identified by MALDI-TOF MS, which was successfully treated with ampicillin followed by linezolid. Furthermore, we conducted a literature review on bacteremia caused by $E$. hirae.

\section{Case presentation}

A 57-year-old male with a history of neurogenic bladder caused by cerebral palsy presented to our emergency department with fever and chills that had persisted for 3 days. He had a history of alcoholic cirrhosis classified as Child-Pugh class $C$ treated with rifaximin, lactulose, and branched-chain amino acid supplementation. The patient reported daily consumption of $500 \mathrm{~mL}$ of Shochu (a traditional Japanese distilled spirit). He had no allergies or significant family history. He was unemployed and denied any recent contact with animals. The patient was diagnosed with a urinary tract infection at a nearby clinic and was prescribed oral cefcapene 2 days before admission. The patient was conscious on admission with a Glasgow Coma Scale of E4V5M6, body temperature of $36.9^{\circ} \mathrm{C}$, blood pressure of $104 / 52 \mathrm{mmHg}$, pulse rate of $82 / \mathrm{min}$, respiratory rate of $20 / \mathrm{min}$, and oxygen saturation of $95 \%$ on room air. On physical examination, tenderness of the right costovertebral angle was noted. Laboratory findings revealed a normal white blood cell (WBC) count of $6,000 / \mu \mathrm{L}$, hemoglobin level of $12.3 \mathrm{~g} / \mathrm{dL}$, platelet count of $48,000 / \mu \mathrm{L}$, creatinine level of $0.92 \mathrm{mg} / \mathrm{dL}$, serum albumin level of $2.9 \mathrm{~g} / \mathrm{dL}$, total bilirubin level of $2.7 \mathrm{mg} /$ $\mathrm{dL}$, and C-reactive protein level of $13 \mathrm{mg} / \mathrm{dL}$. Urinalysis showed protein $2+$, occult blood $2+$, and WBC $2+$. Urine Gram staining revealed gram-positive chains with phagocytosis. Contrast-enhanced computed tomography of the abdomen revealed mild swelling of the kidneys, increased surrounding fat tissue density, and a dull edge and uneven surface of the liver (Fig. 1). We first administered $1 \mathrm{~g}$ of intravenous (IV) ceftriaxone every $24 \mathrm{~h}$. On day 2, we added $2 \mathrm{~g}$ of IV ampicillin every $4 \mathrm{~h}$ because streptococci were cultured from blood and urine samples obtained on admission (BacT/ ALERT FA Plus, BacT/ALERT 3D [bioMérieux Inc.]). On day 4, a transthoracic echocardiogram revealed no evidence of infective endocarditis. On day 5 , final culture results revealed $E$. hirae by matrix-assisted laser desorption ionization-time of flight mass spectrometry (MALDI-TOF MS) (MALDI Biotyper [Bruker Daltonics]) and VITEK2 Compact (bioMérieux Inc.). The minimum inhibitory concentrations measured by MicroScan WalkAway 96 Plus and PC1J panel(Beckman Coulter Inc.) for this strain were as follows: penicillin $\mathrm{G} 0.25 \mu \mathrm{g} / \mathrm{mL}$, ampicillin $0.25 \mu \mathrm{g} / \mathrm{mL}$, vancomycin $1 \mu \mathrm{g} / \mathrm{mL}$, levofloxacin $\leq 0.5 \mu \mathrm{g} / \mathrm{mL}$, teicoplanin $\leq 2 \mu \mathrm{g} /$ $\mathrm{mL}$, and linezolid $2 \mu \mathrm{g} / \mathrm{mL}$ (Table 1). We switched to ampicillin IV ( 2 g every $6 \mathrm{~h}$ ). Blood cultures performed on day 5 were negative. Because his low-grade

Table 1 Antimicrobial susceptibility of the Enterococcus hirae isolated from blood culture in this case

\begin{tabular}{lll}
\hline Antimicrobials & MIC $(\boldsymbol{\mu g} / \mathbf{m L})$ & Susceptibility $^{\mathbf{a}}$ \\
\hline Penicillin G & 0.25 & N/A \\
Ampicillin & 0.25 & Susceptible \\
Vancomycin & 1 & Susceptible \\
Levofloxacin & $\leq 0.5$ & Susceptible \\
Teicoplanin & $\leq 2$ & Susceptible \\
Linezolid & 2 & Susceptible \\
\hline
\end{tabular}

MIC Minimal inhibitory concentration

${ }^{\text {a }}$ Based on the European Committee on Antimicrobial Susceptibility Testing (EUCAST) Clinical Breakpoints v.11.0, for Enterococcus spp.[15]


Fig. 1 Contrast-enhanced computed tomographic images revealing heterogeneous enhancement of both kidneys in $\mathbf{A}$, and a liver with a blunt edge and irregular surface in $\mathbf{B}$ 
fever persisted, we switched to oral linezolid $600 \mathrm{mg}$ every $12 \mathrm{~h}$ on day 11 , considering possible drug fever. Thereafter, the patient defervesced and was discharged on day 15. He completed a course of oral linezolid for 3 weeks in total, and his condition resolved without any relapse of symptoms at the 10-month follow-up.

\section{Methods of literature review}

Two authors independently reviewed the titles and abstracts of database records, retrieved full texts for eligibility assessment, and extracted data from these case reports. We ran searches on the PubMed database (up to May 2020) using the keywords (("Enterococcus hirae"[Mesh]) OR ("Enterococcus hirae"[TW]) OR (hirae[TIAB])) AND ((Bacteremia[MH]) OR (bacteremia*[TIAB] OR bacteraemia*[TIAB]))) OR ((("Enterococcus hirae"[Mesh]) OR ("Enterococcus hirae"[TW]) OR (hirae[TIAB])) AND Humans[MH]), and the Embase database using the keywords (('bacteremia'/exp OR 'gram negative sepsis'/exp OR bacteraemia* OR bacteremia*) AND ('enterococcus hirae'/ exp OR hirae)) OR (('enterococcus hirae'/exp OR hirae) AND [humans]/lim). PubMed and Embase searches generated 170 and 229 articles, respectively. Of these, 158 and 218 articles from PubMed and Embase, respectively, were excluded because they were not case reports (Fig. 2). We searched Google Scholar and identified eight more human cases. Manuscripts not written in English were excluded. Finally, we reviewed 21 articles that included 31 strains from human sources.

\section{Discussion and conclusion}

Enterococcus hirae was first identified by Farrow et al. in 1985 [16]. It has been reported that although animal species such as chickens, rats, birds, and cats are commonly found to be infected [1,2], human infections are relatively rare [17]. Only 31 human cases of $E$. hirae have been reported (Table 2). Of these, urinary tract infections $[3-5,12,14,18]$, biliary tract infections $[5,12]$, and infective endocarditis [6-11] accounted for the majority of cases, with catheter-related bloodstream infections [12, 19], peritonitis [20, 21], splenic abscess [22], and pneumonia [17] also being reported. Patients were predominantly male $(n=20,64.5 \%)$, similar to predominance in infections caused by other Enterococcus spp. [23], Furthermore, no age trend was observed (median: 63 years) [23]. The common underlying diseases were diabetes mellitus $(\mathrm{n}=12,39 \%)$, liver cirrhosis $(\mathrm{n}=4,13 \%)$, and chronic kidney disease $(\mathrm{n}=4,13 \%)$. Occurrence of diabetes mellitus and liver cirrhosis was consistent with previous reports of Enterococcus spp. Malignant tumors were found to be less common [23]. This case of a middle-aged male with underlying alcoholic cirrhosis and chronic kidney disease was consistent with the trend uncovered in the literature review.

In this review, one case of death due to biliary tract infection caused by $E$. hirae was reported [12]. The mortality rate $(\mathrm{n}=1,3 \%)$ from $E$. hirae infection was similar






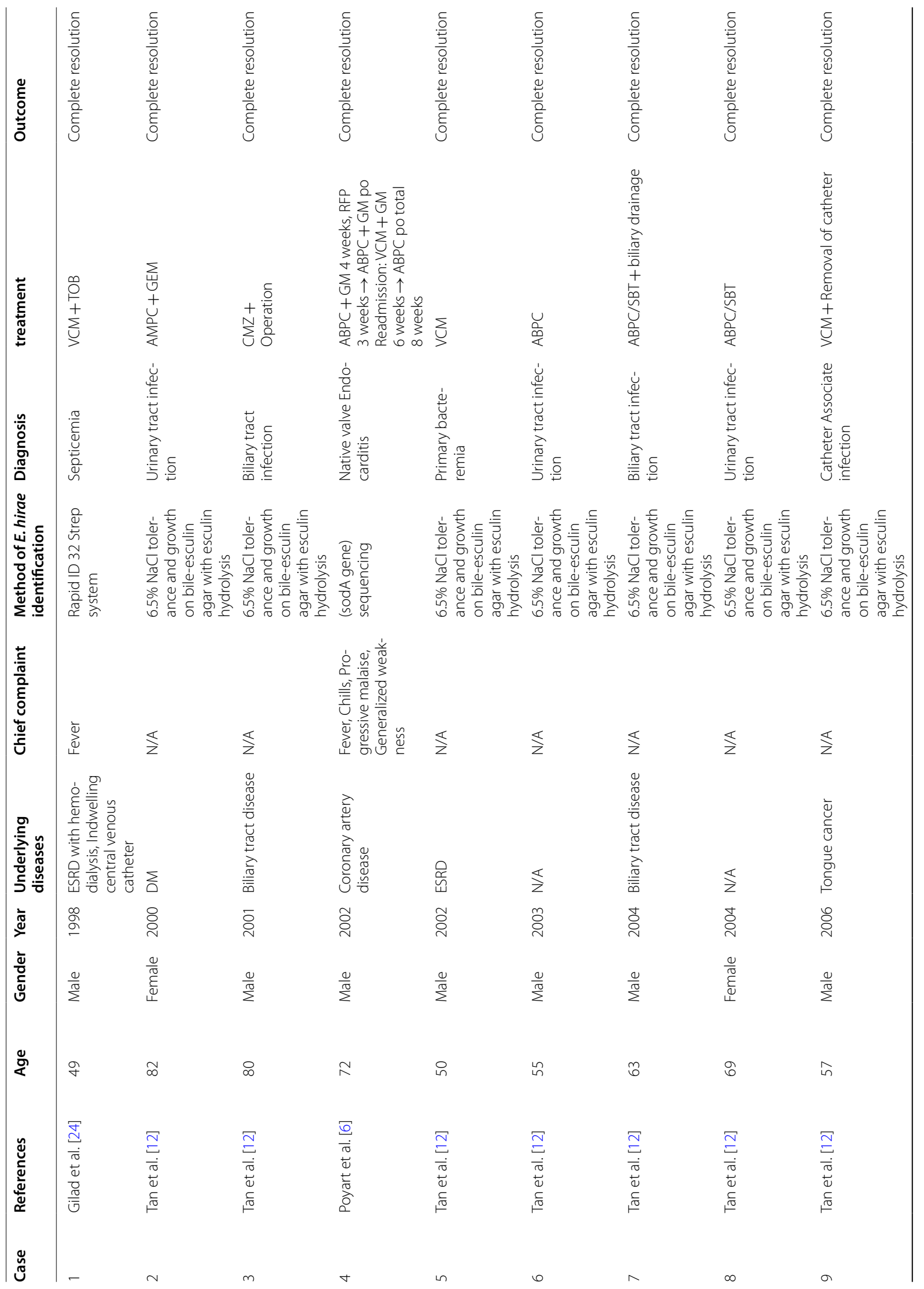




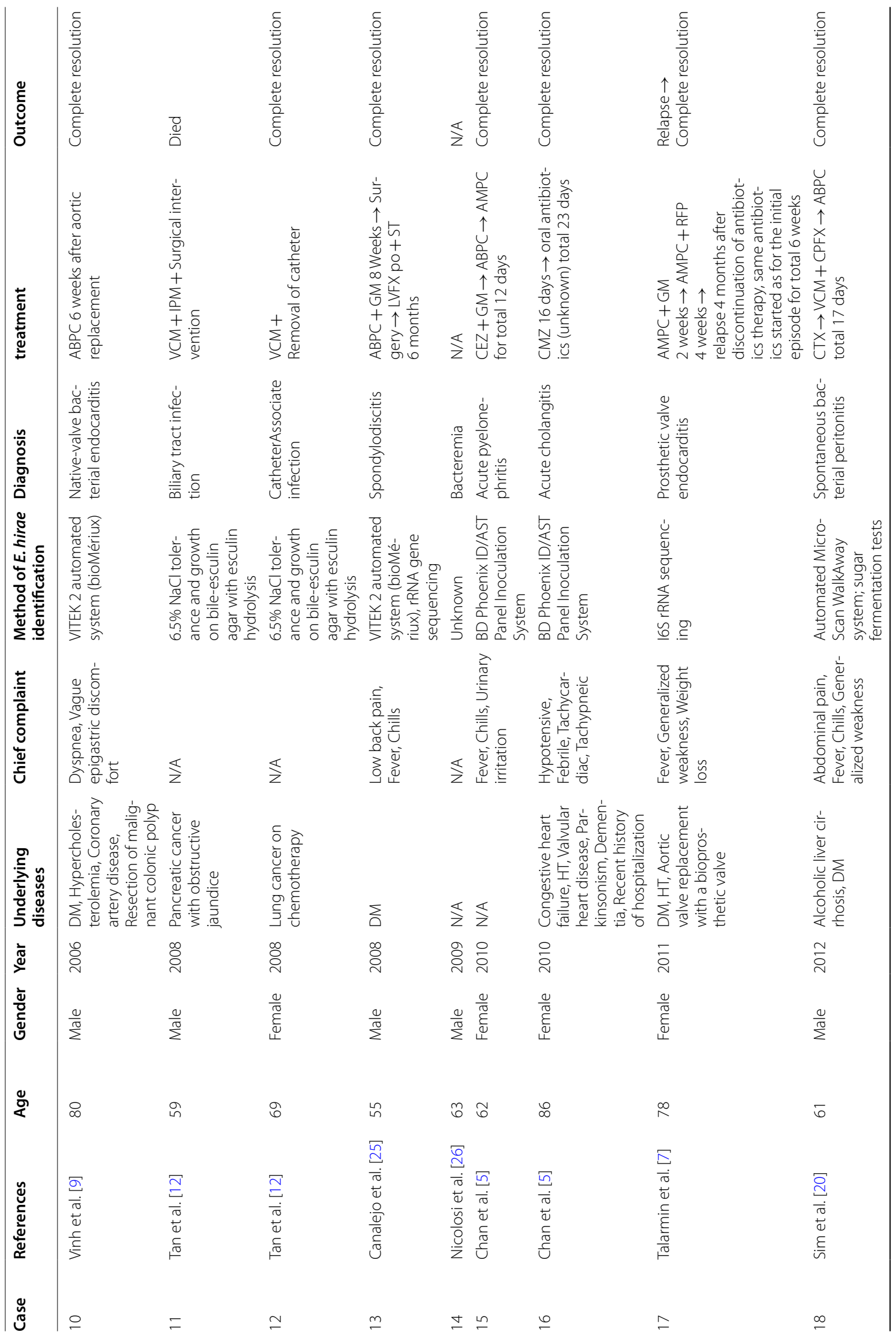









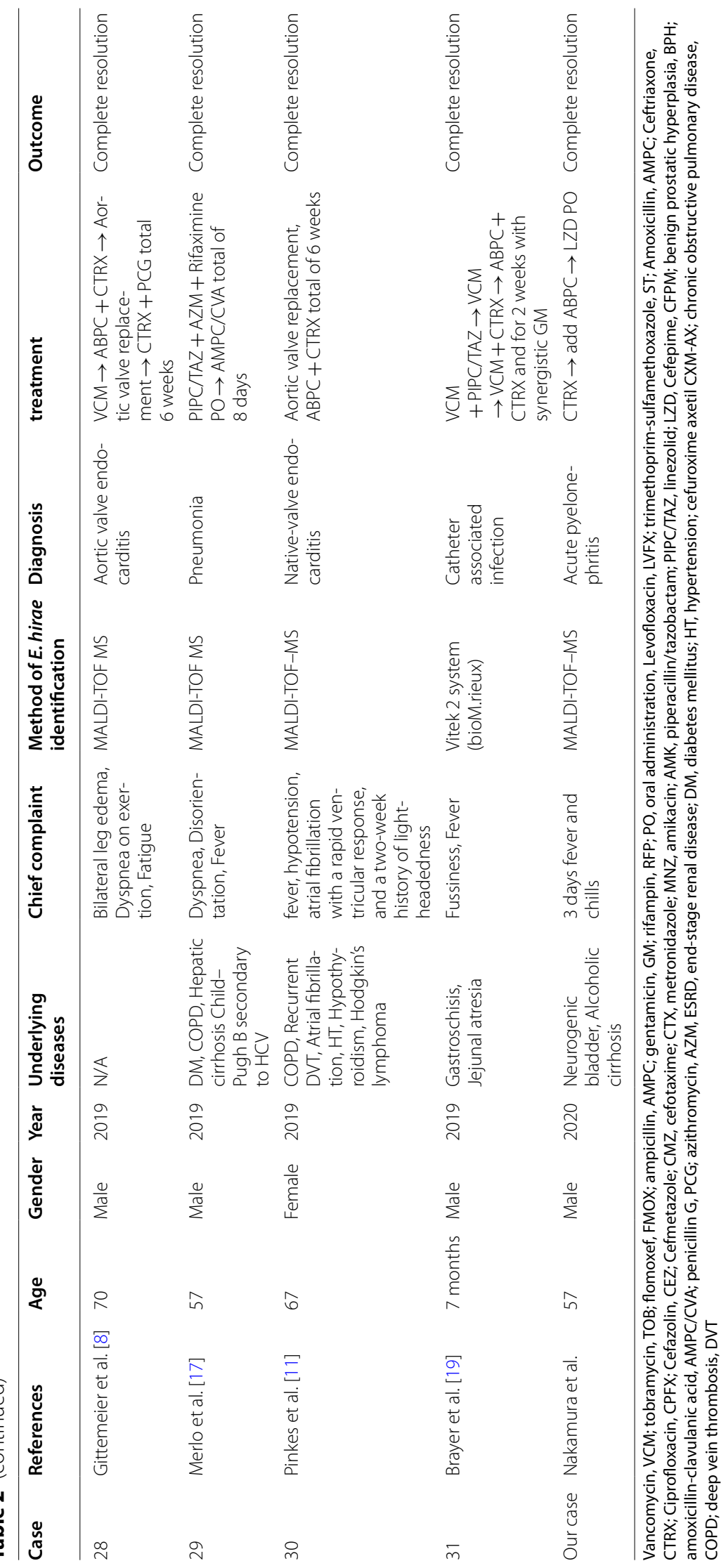


to or lower than that of other Enterococcus spp. infections (23\%) [23]. However, the accumulation of E. hirae infections warrants accurate evaluation.

Three cases of $E$. hirae infection recurred during treatment $[6,7,21]$, and two of the three recurrent cases involved infective endocarditis. In a report comparing 3308 cases of infective endocarditis caused by nonEnterococcus spp. with 516 cases of infective endocarditis caused by Enterococcus spp. collected prospectively from
35 centers in Spain, recurrence was significantly higher in cases of infective endocarditis caused by Enterococcus spp. (3.5\% vs. $1.7 \%)$ [28]. There were nine reported cases of $E$. hirae urinary tract infections with no recurrences or deaths.

The susceptibility of $E$. hirae to antimicrobial agents is similar to that of E. faecalis, which is susceptible to penicillin. Table 3 shows the antimicrobial susceptibility of E. hirae infections in humans. Although some

Table 3 Summary of antimicrobial susceptibility in the previously reported human cases with Enterococcus hirae

\begin{tabular}{|c|c|c|c|}
\hline Case & Sensitive & Resistance & Supplement \\
\hline 1 & ABPC, VCM, IPM GM & N/A & No beta-lactamase activity \\
\hline 2 & N/A & $\mathrm{N} / \mathrm{A}$ & \\
\hline 3 & N/A & $\mathrm{N} / \mathrm{A}$ & \\
\hline 4 & ABPC, VCM, TEIC, CP & CLDM, EM, RFP, TC & Low-level resistance to SM, KM, GM \\
\hline 5 & N/A & N/A & \\
\hline 6 & N/A & N/A & \\
\hline 7 & N/A & $\mathrm{N} / \mathrm{A}$ & \\
\hline 8 & N/A & $\mathrm{N} / \mathrm{A}$ & \\
\hline 9 & $\mathrm{~N} / \mathrm{A}$ & $\mathrm{N} / \mathrm{A}$ & \\
\hline 10 & ABPC, PCG, CP, CPFX, OFLX, LVFX, TC, VCM & CLDM, NFLX & $\begin{array}{l}\text { No evidence of high-level aminoglycoside } \\
\text { resistance to GM or SM } \\
\text { Intermediate susceptibility to EM, NTF }\end{array}$ \\
\hline 11 & N/A & $\mathrm{N} / \mathrm{A}$ & \\
\hline 12 & N/A & $\mathrm{N} / \mathrm{A}$ & \\
\hline 13 & N/A & CLDM, Cephalosporins & \\
\hline 14 & $E M, C P, L Z D, V C M$ & RFP & Intermediate susceptibility to ABPC, DOXY \\
\hline 15 & ABPC, TEIC, VCM, high dose GM & OFLX, GM & \\
\hline 16 & ABPC, TEIC, VCM, high dose GM & OFLX, GM & \\
\hline 17 & ABPC, MFLX, VCM, TEIC, EM, RFP & CLDM, FOS & Low-level resistance to SM, KM, GM \\
\hline 18 & $\begin{array}{l}\text { ABPC, VCM, TEIC, EM, TC } \\
\text { high-level SM and GM }\end{array}$ & $N / A$ & \\
\hline 19 & AMPC & Cephalosporins & \\
\hline 20 & N/A & N/A & \\
\hline 21 & ABPC, VCM, TEIC, LZD, TC & CPFX & No high-level resistance to GM \\
\hline 22 & ABPC, VCM, CPFX & N/A & \\
\hline 23 & high-level GM and KM, ABPC, LZD, CPFX, Nitrofuran, VCM & ST & \\
\hline 24 & AMPC/CVA, PIPC/TAZ & CXM-AX, NTF & \\
\hline 25 & VCM & N/A & \\
\hline 26 & $\begin{array}{l}\text { ABPC, ABPC/SBT, CPFX, EM, high-level GM, IPM, LVFX, LZD, } \\
\text { NFLX, PCG, QPR/DPR, high-level SM, TEIC, TC, VCM, TGC }\end{array}$ & none & intermediate susceptibility to NTF \\
\hline 27 & $\begin{array}{l}\text { ABPC, ABPC/SBT, CPFX, EM, high-level GM, IPM, LVFX, LZD, } \\
\text { NFLX, PCG, QPR/DPR, high-level SM, TEIC, TC, VCM, TGC }\end{array}$ & none & intermediate susceptibility to NTF \\
\hline 28 & N/A & $N / A$ & \\
\hline 29 & ABPC, IPM, GM, CPFX, LVFX, VCM, TEIC, ST, LZD, TGC & $\mathrm{N} / \mathrm{A}$ & \\
\hline 30 & ABPC, AMPC, VCM & $\mathrm{N} / \mathrm{A}$ & It demonstrated synergy with GM and SM \\
\hline 31 & ABPC, VCM, high-level GM & $\mathrm{N} / \mathrm{A}$ & \\
\hline 32 & ABPC, PCG, VCM, LVFX, TEIC, LZD & none & \\
\hline
\end{tabular}

Ampicillin, ABPC; Vancomycin, VCM; Imipenem, IPM; Gentamicin, GM; Teicoplanin, TEIC; Chloramphenicol, CP; Clindamycin, CLDM; Erythromycin, EM; Rifampin, RFP; Streptomycin, SM; Kanamycin, KM; Penicillin G, PCG; CPFX, Ciprofloxacin; Levofloxacin, LVFX; Tetracycline; TC; Linezolid, LZD; doxycycline, DOXY; Moxifloxacin, MFLX; Amoxicillin, AMPC; Amoxicillin-clavulanic acid, AMPC/CVA; Piperacillin/tazobactam, PIPC/TAZ; Ampicillin sulbactam, ABPC/SBT; trimethoprim-sulfamethoxazole, ST; Cefuroxime axetil, CXM-AX; Norfloxacin, NFLX; Quinupristin/Dalfopristin, QPR/DPR; Fosfomycin, FOS; Tigecycline, TGC; Ofloxacin OFLX; Nitrofurantoin, NTF 
reports have reported high resistance to gentamicin [29], of the 21 antimicrobial-susceptible cases in this review, only four (19\%) were gentamicin-resistant, and high-level gentamicin resistance cases were not reported. The relatively low mortality and antimicrobial resistance suggest that $E$. hirae is more similar to $E$. faecalis than E. faecium. In the present case, the patient could not tolerate ampicillin due to drug allergy and was successfully treated with linezolid after confirming susceptibility. Resistance to clindamycin and gentamicin has been reported repeatedly, and the possibility of resistance should be considered when these drugs are used. The accumulation of human clinical data is warranted to generate an accurate evaluation.

Matrix assisted laser desorption ionization-time of flight mass spectrometry (MALDI-TOF MS) was developed in the 1980s and was accurate in $80-95 \%$ of bacterial isolates [13]. Species-level identifications have been obtained and have been widely used in recent years [13]. A study validated the accuracy of MALDI-TOF MS for the identification of Enterococcus spp. compared with the gold standard rpoA gene sequencing method for the identification of bacteria of environmental origin. The occurrence of Enterococcus spp., including E. hirae, in wild birds was correctly identified by MALDI-TOF MS [30]. Before the advent of MALDI-TOF-MS, E. hirae may have been underdiagnosed because of the limitations of the diagnostic method [3]. This review found that there has been an increase in reporting of $E$. hirae since 2015 following the advent of MALDI-TOF MS.

Enterococcus hirae is a newly recognized causative pathogen of urinary tract infections, especially in patients with underlying diseases. Clinical data such as risk factors, clinical manifestations, and antimicrobial susceptibility are lacking, and more cases should be accumulated following accurate identification.

In summary, the number of $E$. hirae infections reported has increased following the development of MALDI-TOF MS. Although E. hirae may have a low virulence, as do other enterococci, clinicians need to consider $E$. hirae as a causative pathogen of urinary tract infection.

\footnotetext{
Abbreviations

E. hirae: Enterococcus hirae; IV: Intravenous; MALDI-TOF MS: Matrix-assisted laser desorption ionization-time of flight mass spectrometry; WBC: White blood cell.

\section{Acknowledgements}

Not applicable.

\section{Authors' contributions}

The manuscript was seen and approved by all the authors and is not under consideration elsewhere. All the authors contributed to the work in this report. TN collected clinical data and wrote the initial draft of the manuscript. $\mathrm{TN}, \mathrm{KI}$, and FK performed the review of the literature. $\mathrm{KI}, \mathrm{TM}, \mathrm{YU}$, and $\mathrm{NM}$ supervised and edited the manuscript. All authors read and approved the final manuscript.
}

\section{Funding}

There were no sources of funding used in the conception, composition, editing, or submission of this manuscript.

Availability of data and materials

Not applicable.

\section{Declarations}

Ethics approval and consent to participate

Not applicable.

\section{Consent for publication}

Written informed consent was obtained from the patient for publication of this case report and any accompanying images.

\section{Competing interests}

The authors declare that they have no competing interests.

\section{Author details}

${ }^{1}$ Department of Infectious Diseases, St. Luke's International Hospital, 9-1, Akashi-cho, Chuo-ku, Tokyo, Japan. ${ }^{2}$ St. Luke's International University Library, Tokyo, Japan. ${ }^{3}$ Department of Clinical Laboratory, St. Luke's International Hospital, Tokyo, Japan. ${ }^{4}$ Department of Microbiology, Faculty of Medicine, Juntendo University, Tokyo, Japan. ${ }^{5}$ Department of General Medicine, Faculty of Medicine, Juntendo University, Tokyo, Japan.

Received: 10 March 2021 Accepted: 16 September 2021

Published online: 23 September 2021

\section{References}

1. Etheridge ME, Yolken RH, Vonderfecht SL. Enterococcus hirae implicated as a cause of diarrhea in suckling rats. J Clin Microbiol. 1988;26:1741-4.

2. Devriese LA, Haesebrouck F. Enterococcus hirae in different animal species. Vet Rec. 1991;129:391-2.

3. Pãosinho A, Azevedo T, Alves JV, et al. Acute pyelonephritis with bacteremia caused by Enterococcus hirae: a rare infection in humans. Case Rep Infect Dis. 2016;2016:4698462.

4. Lee GH, Lee HW, Lee YJ, Park BS, Kim YW, Park S. Acute pyelonephritis with Enterococcus hirae and literature review. Urogenit Tract Infect. 2017;12:49-53.

5. Chan TS, Wu MS, Suk FM, et al. Enterococcus hirae-related acute pyelonephritis and cholangitis with bacteremia: an unusual infection in humans. Kaohsiung J Med Sci. 2012;28:111-4.

6. Poyart C, Lambert T, Morand P, Abassade P, Quesne G, Baudouy Y, et al. Nativevalve endocarditis due to Enterococcus hirae. J Clin Microbiol. 2002;40:2689-90.

7. Talarmin JP, Pineau S, Guillouzouic A, Boutoille D, Giraudeau C, Reynaud A, et al. Relapse of Enterococcus hirae prosthetic valve endocarditis. J Clin Microbiol. 2011;49:1182-4.

8. Ebeling CG, Romito BT. Aortic valve endocarditis from Enterococcus hirae infection. Baylor Univ Med Cent Proc. 2019;32:249-50.

9. Vinh DC, Nichol KA, Rand F, Embil JM. Native-valve bacterial endocarditis caused by Lactococcus garvieae. Diagn Microbiol Infect Dis. 2006;56:91-4.

10. Anghinah R, Watanabe RG, Simabukuro MM, Guariglia C, Pinto LF, Gonçalves DC. Native valve endocarditis due to Enterococcus hirae presenting as a neurological deficit. Case Rep Neurol Med. 2013;2013:636070.

11. Pinkes ME, White C, Wong CS. Native-valve Enterococcus hirae endocarditis: a case report and review of the literature. BMC Infect Dis. 2019;19:891.

12. Tan CK, Lai CC, Wang JY, et al. Bacteremia caused by non-faecalis and non-faecium enterococcus species at a Medical center in Taiwan, 2000 to 2008. J Infect. 2010;61:34-43.

13. Bizzini A, Greub G. Matrix-assisted laser desorption ionization time-offlight mass spectrometry, a revolution in clinical microbial identification. Clin Microbiol Infect. 2010;16:1614-9.

14. Bourafa N, Loucif L, Boutefnouchet N, Rolain JM. Enterococcus hirae, an unusual pathogen in humans causing urinary tract infection in a patient 
with benign prostatic hyperplasia: first case report in Algeria. New Microbes New Infect. 2015;8:7-9.

15. European Committee on Antimicrobial Susceptibility Testing (EUCAST) website. https://www.eucast.org/clinical_breakpoints/

16. Farrow JA, Collins MD. Enterococcus hirae, a new species that includes amino acid assay strain NCDO 1258 and strains causing growth depression in young chickens. Int J Syst Bacteriol. 1985;35:73-5.

17. Merlo J, Bustamante G, Llibre JM. Bacteremic pneumonia caused by Enterococcus hirae in a subject receiving regorafenib. Enferm Infecc Microbiol Clin. 2019;38:226-9.

18. Brulé N, Corvec S, Villers D, Guitton C, Bretonnière C. Life-threatening bacteremia and pyonephrosis caused by Enterococcus hirae. Med Mal Infect. 2013:43:401-2.

19. Brayer S, Linn A, Holt S, Ellery K, Mitchell S, Williams J. Enterococcus hirae bacteremia in an infant: case report and review of the literature. J Pediatric Infect Dis Soc. 2019;8:571-3.

20. Sim JS, Kim HS, Oh KJ, Park MS, Jung EJ, Jung YJ, et al. Spontaneous bacterial peritonitis with sepsis caused by Enterococcus hirae. J Korean Med Sci. 2012;27:1598-600.

21. Atas DB, Aykent B, Asicioglu E, Arikan H, Velioglu A, Tuglular S, et al. Peritoneal dialysis-related peritonitis with an unexpected micro-organism: Enterococcus hirae. Med Sci Int Med J. 2016;6:120-1.

22. Alfouzan W, Al-Sheridah S, Al-Jabban A, Dhar R, Al-Mutairi AR, Udo E. A case of multiple splenic abscesses due to Enterococcus hirae. JMM Case Rep. 2014;1:1-4

23. Billington EO, Phang SH, Gregson DB, et al. Incidence, risk factors, and outcomes for Enterococcus spp. blood stream infections: a populationbased study. Int J Infect Dis. 2014;26:76-82.
24. Gilad J, Borer A, Riesenberg K, Peled N, Shnaider A, Schlaeffer F. Enterococcus hirae septicemia in a patient with end-stage renal disease undergoing hemodialysis. Eur J Clin Microbiol Infect Dis. 1998;17:576-7.

25. Canalejo E, Ballesteros R, Cabezudo J, García-arata MI, Moreno J. Bacteraemic spondylodiscitis caused by Enterococcus hirae. Eur J Clin Microbiol Infect Dis. 2008;27:613-5.

26. Nicolosi D, Nicolosi VM, Cappellani A, Nicoletti G, Blandino G. Antibiotic susceptibility profiles of uncommon bacterial species causing severe infections in Italy. J Chemother. 2009;21:253-60.

27. Dicpinigaitis PV, de Aguirre M, Divito J. Enterococcus hirae Bacteremia associated with acute pancreatitis and septic shock. Case Rep Infect Dis. 2015;2015:123852.

28. Pericàs JM, Llopis J, Muñoz $\mathrm{P}$, et al. A contemporary picture of enterococcal endocarditis. J Am Coll Cardiol. 2020;75:482-94.

29. Mangan MW, McNamara EB, Smyth EG. Storrs MJ (1997) Molecular genetic analysis of high-level gentamicin resistance in Enterococcus hirae. J Antimicrob Chemother. 1997:40:377-82.

30. Stępień-pyśniak D, Hauschild T, Różański P, Marek A. MALDI-TOF mass spectrometry as a useful tool for identification of spp. from wild birds and differentiation of closely related species. J Microbiol Biotechnol. 2017;27:1128-37.

\section{Publisher's Note}

Springer Nature remains neutral with regard to jurisdictional claims in published maps and institutional affiliations.
Ready to submit your research? Choose BMC and benefit from:

- fast, convenient online submission

- thorough peer review by experienced researchers in your field

- rapid publication on acceptance

- support for research data, including large and complex data types

- gold Open Access which fosters wider collaboration and increased citations

- maximum visibility for your research: over 100M website views per year

At BMC, research is always in progress.

Learn more biomedcentral.com/submissions 